\title{
Model Based Anticontrol of Discrete-Time Systems
}

\author{
Ömer Morgül \\ Bilkent University \\ Dept. of Electrical and Electronics Engineering \\ 06800, Bilkent, Ankara, Turkey \\ morguleee.bilkent.edu.tr
}

\begin{abstract}
We will consider a model-based approach for the anticontrol of some discrete-time systems. We first assume the existence of a chaotic model in an appropriate form. Then by using an appropriate control input we try to match the
\end{abstract} controlled system with the chaotic system model.

Key Words : Chaotic Systems, Chaos Control, Chaotification, Anticontrol, Chaos Synchronization.

\section{INTRODUCTION}

The analysis and control of chaotic behaviour in dynamical systems has been investigated by many researchers in various disciplines on recent years. Among the vast amount of works already published in the literature, interested reader may consult to e.g. various survey papers such as [5], [7], to research monographs such as [2] , [6], and to a bibliography [1].

While in majority of the works in the area of chaos control the main aim is the suppression of chaotic behaviour, see e.g. [2], [7], the opposite approach, i.e. to retain the chaotic behaviour, or even to force a regular behaviour into a chaotic one, has also received considerable interest. This problem is known as "anticontrol" or "chaotification", and has a great potential for application in diverse fields, see [9] and the references therein. Various feedback schemes, mostly for discrete-time systems are available in the literature for the anticontrol of such systems, see e.g. [3], [4].

In this work, we will consider a model-based approach for the anticontrol of some discrete-time systems. We first assume the existence of a chaotic model in an appropriate form. Then by using an appropriate control input we try to match the controlled system with the chaotic system model. We prove that :

$i$ : any controllable linear time-invariant system can be chaotified with an appropriate input,

$i i$ : this approach could be generalized to a class of nonlinear systems.

We also address to the question of the existence of model chaotic systems. We propose a simple procedure to generate such chaotic models in arbitrary dimension. We note that this approach could also be applied to the anticontrol of continuous-time systems, see [8].

\section{Problem Statement}

We will first consider the linear systems. Consider the system given below :--

$$
x(k+1)=A x(k)+B u(k), y(k)=C x(k),
$$

where $x \in \mathbf{R}^{n}, A \in \mathbf{R}^{n \times n}$ is a constant matrix, $B, C^{T} \in \mathbf{R}^{n}$ are constant vectors, here superscript $T$ denotes transpose, $u$ is the (scalar) control input and $y$ is the (scalar) output, which is assumed to be measurable, and $k=0,1,2, \ldots$ is the discrete time index. For this system, we pose the following problem

Problem : Find a feedback law $u(k)=g(x(k))$, where $g$ : $\mathbf{R}^{n} \rightarrow \mathbf{R}$ is an appropriate function, such that the resulting closed-loop system exhibits chaotic behaviour.

Let $p(\lambda)$ be the characteristic polynomial of $A$ given by (1), which is given as follows : $p(\lambda)=\operatorname{det}(\lambda I-A)=\lambda^{n}+$ $\alpha_{1} \lambda^{n-1}+\ldots+\alpha_{n-1} \lambda+\alpha_{n}$.

It can be easily shown that if the system given by (1) is controllable then by using an appropriate coordinate transformation $z=R x$, it can be transformed into the form

$$
\left.\begin{array}{c}
z_{i}(k+1)=z_{i+1}(k) \quad i=1,2, \ldots, n-1 \\
z_{n}(k+1)=-\sum_{i=1}^{n} \alpha_{n+1-i} z_{i}(k)+u(k)
\end{array}\right\}
$$

\section{MOdel BASEd SCHEME}

Let us assume that our model chaotic system is given as follows :

$$
\left.\begin{array}{l}
w_{i}(k+1)=w_{i+1}(k) \quad i=1,2, \ldots, n-1 \\
w_{n}(k+1)=f\left(w_{1}(k), w_{2}(k), \ldots, w_{n}(k)\right)
\end{array}\right\}
$$

where $f: \mathbf{R}^{n} \rightarrow \mathbf{R}$ is an appropriate function. In the section 4 , we will propose a simple scheme to generate chaotic systems of this form for arbitrary dimension $n>1$.

Our anti-control scheme is based on matching the system given by (2) with the model chaotic system given by (3) by using an appropriate control input $u(k)$. Hence, to achieve this goal, we may choose $u(k)$ as

$$
u(k)=f(z(k))+\sum_{i=1}^{n} \alpha_{n+1-i} z_{i}(k) \quad \vdots
$$

Obviously, by using (4) we can transform (2) into the chaotic system given by (3).

- The anticontrol scheme given above can also be applied to a class of nonlinear systems which can be transformed into 
the following controllable form :

$$
\begin{gathered}
z_{i}(k+1)=z_{i+1}(k) \quad i=1,2, \ldots, n-1 \\
z_{n}(k+1)=-\sum_{i=1}^{n} \alpha_{i} z_{i}(k)+\gamma(z(k))+\beta(z(k)) u(k)
\end{gathered}
$$

where $\gamma(z(k))$ and $\beta(z(k))$ are appropriate functions.

\section{Model ChaOtic Systems}

In the sequel we will present a simple scheme to generate chaotic systems of the form given by (3) in arbitrary dimension $n$. Note that for $n=1$, the required form reduces to $w(k+1)=f(w(k))$, and there are many one-dimensional chaotic systems in this form. Now assume that there exists a chaotic system of the form given by (3) for $n \geq 1$. We will present a simple scheme to generate a chaotic system of the same form for dimension $n+1$. Consider the following system :

$$
\left.\begin{array}{c}
w_{i}(k+1)=w_{i+1}(k) \quad i=1,2, \ldots, n-1 \\
w_{n}(k+1)=f\left(w_{1}(k), w_{2}(k), \ldots, w_{n}(k)\right)+z(k) \\
z(k+1)=\rho z(k)
\end{array}\right\}
$$

where $|\rho|<1$ is an arbitrary real number. Obviously, $z(k)=$ $\rho^{k} z(0) \rightarrow 0$ as $k \rightarrow \infty$, hence the first $n$ equations of (5) and (3) are asymptotically the same. Therefore if (3) has a-globally attractive chaotic attractor, so does (5). On the other hand, if (3) has only locally attractive chaotic attractor, which is structurally stable, then so does (5) provided that $|z(0)|$ is sufficiently small.

To transform (5) into the form (3), let us define the variable $w_{n+1}$ as follows :

$$
w_{n+1}(k)=f\left(w_{1}(k), w_{2}(k), \ldots, w_{n}(k)\right)+z(k) .
$$

Hence, from (5) we have $w_{n}(k+1)=w_{n+1}(k)$. By using (6), (5) can be rewritten as follows :

$$
\left.\begin{array}{c}
w_{i}(k+1)=w_{i+1}(k) \quad i=1,2, \ldots, n \\
w_{n+1}(k+1)=F\left(w_{1}(k), w_{2}(k), \ldots, w_{n+1}(k)\right)
\end{array}\right\}
$$

where $F$ is given as :

$$
\begin{aligned}
F(w(k))= & f\left(w_{2}(k), w_{3}(k), \ldots, w_{n+1}(k)\right)+\rho w_{n+1}(k) \\
& -\rho f\left(w_{1}(k), w_{2}(k), \ldots, w_{n}(k)\right)
\end{aligned}
$$

\section{CONCLUSION}

In this paper, we considered a model-based approach to the anticontrol of some discrete-time systems. Our aim was to generate a chaotic behaviour which is determined by a chaotic model, by means of an appropriate control input. To achieve this task, we assumed the existence of a reference model in an appropriate form which exhibits chaotic behaviour. Then we determined an appropriate control input to match the dynamics of the system to be controlled with that of the model chaotic system. We proved that $: i$ : any controllable linear time-invariant system can be chaotified with an appropriate input, $i i$ : this approach could be generalized to a class of nonlinear systems. We proposed a simple procedure to generate such chaotic models in arbitrary dimension.

\section{REFERENCES}

[1] Chen, G. "Control and synchronization of chaotic systems, (a bibliography)", ftp.egr.uh.edu/pub/TeX/chaos.tex loginname: anonymous, password: your e-mail address.

[2] Chen, G., \& Dong, X., From Chaos to Order: Methodologies, Perspectives and Applications, (World Scientific, Singapore), 1998.

[3] Chen, G., \& Lai, D., "Feedback control of Lyapunov exponents for discrete-time dynamical systems," Int. $J$. Bifurcation and Chaos, 6, pp. 1341-1349, 1996.

[4] Chen, G., \& Lai, D., "Feedback anticontrol of discrete chaos," Int. J. Bifurcation and Chaos, 8, pp. 1585-1590, 1998.

[5] Chen, G., \& Moiola, J.L., "An overview of bifurcation, chaos, and nonlinear dynamics in nonlinear systems," J. Franklin Inst., 331B, pp. 819-858, 1994.

[6] Fradkov, A.L., \& Pogromsky, A.Y., Introduction to control of oscillations and chaos, (World Scientific, Singapore), 1998.

[7] Fradkov, A.L., \& Evans, R.J., "Control of chaos : Survey 1997-2000," Proceedings of IFAC 2002 World Congress, July 2002, Barcelona, Spain, 2002.

[8] Morgül, Ö. , "A model based scheme for anticontrol of some chaotic systems," accepted for publication in Int. J. of Bifurcation and Chaos, 2002.

[9] Wang, X.F., \& Chen, G., "Chaotifying a stable LTI system by tiny feedback control," IEEE Trans. Circuits Syst. 1, 47, pp. 410-415, 2000. 